\title{
Sevoflurane anesthesia alters cognitive function by activating inflammation and cell death in rats
}

\author{
RONG-SHENG CUI, KAI WANG and ZHONG-LI WANG \\ Department of Anesthesia, People's Hospital of Rizhao, Rizhao, Shandong 276800, P.R. China \\ Received January 10, 2017; Accepted August 31, 2017
}

DOI: $10.3892 /$ etm.2018.5976

\begin{abstract}
The present study was designed to investigate the effects of sevoflurane inhalation anesthesia on the cognitive function of rats and to investigate the molecular mechanisms mediating this effect. A total of 100 healthy male Sprague-Dawley rats were divided into four groups: i) Control (air inhalation), ii) low-dose (1.5\% sevoflurane inhalation for $2 \mathrm{~h}$ ), iii) high-dose (3\% sevoflurane inhalation for $2 \mathrm{~h}$ ), and iv) nimodipine group (3\% sevoflurane inhalation for $2 \mathrm{~h}+$ nimodipine). Sevoflurane inhalation anesthesia resulted in cognitive dysfunction in a dose-dependent manner. Sevoflurane also upregulated the expression of tumour necrosis factor- $\alpha$ (TNF- $\alpha$ ), interleukin (IL) $-6,-8$, and Caspase- 3 in the hippocampus. The intervention with nimodipine partially recovered the cognitive function and the abnormal expression of TNF- $\alpha$, IL-6, IL-8, and Caspase-3 induced by sevoflurane. The results showed that the cognitive dysfunction caused by sevoflurane inhalation in rats may be related to the activation of inflammatory and apoptotic pathways. The neuroprotective effect of nimodipine suggests that abnormal calcium transport is partially responsible for the sevoflurane toxicity.
\end{abstract}

\section{Introduction}

Owing to the social and economic development of China, patients in need of surgery are currently on the increase. Improved control in intensity and depth has led to use of inhalation anesthesia having advantages over intravenous anesthesia in surgery. Due to its mild irritation, rapid induction, stable maintenance, and rapid revival, sevoflurane has replaced other inhalational anesthetics in surgery and has become a first-line drug (1). However, previous findings have indicated that sevoflurane can lead to postoperative cognitive dysfunction (2), although the evidence for this effect is anecdotal.

Correspondence to: Dr Kai Wang, Department of Anesthesia, People's Hospital of Rizhao, 126 Taian Road, Rizhao, Shandong 276800, P.R. China

E-mail: luqi80419@163.com

Key words: sevoflurane, cognitive function, interleukin, apoptosis protein, nimodipine
Postoperative cognitive dysfunction or delirium refers to changes in cognitive function affecting orientation, thinking, attention, and insight (3), and is one of the most common postoperative complications. Postoperative cognitive dysfunction seriously affects the life of patients, prolongs hospitalization, and increases the costs for medical care, thus bringing additional burden to the patients and their families (4). Therefore, the relationship between postoperative cognitive dysfunction, anesthesia administration, and surgical trauma has increasingly attracted the attention of anesthesia experts and clinicians. Present research has not fully elucidated the specific mechanism by which sevoflurane affects cognitive dysfunction.

Sevoflurane inhalation was utilized in the present study to determine its effects on cognitive function in rats. We analyzed changes in the expression of tumour necrosis factor- $\alpha$ (TNF- $\alpha$ ), interleukin (IL) $-6,-8$, and apoptosis in the hippocampus of rats treated with sevoflurane, providing experimental data for future clinical applications.

\section{Materials and methods}

Animals and grouping. We obtained 100 male Sprague-Dawley rats, 2 months old with an average weight of 250-350 g (Nanjing Junke Biological Engineering Co., Ltd., Nanjing, China). The rats were fed ad libitum and kept in a $12 \mathrm{~h}$ light/dark cycle at $22-24^{\circ} \mathrm{C}$. The rats were randomly divided into 4 groups: i) Control (air inhalation), ii) low-dose (1.5\% sevoflurane inhalation for $2 \mathrm{~h}$ ), iii) high-dose ( $3 \%$ sevoflurane inhalation for $2 \mathrm{~h}$ ), and iv) nimodipine (3.0\% sevoflurane inhalation for $2 \mathrm{~h}+$ nimodipine treatment). Nimodipine is a calcium channel blocker that lowers blood pressure and reduces brain damage from a burst blood vessel (5).

Major instruments. The instruments used in the present study were: Anesthesia machine (Shanghai Yuyan Instruments Co., Ltd., Shanghai, China), anesthesia gas monitor (Harvard Apparatus, Holliston, MA, USA), electronic balance (BS200S; Sartorius AG, Göttingen, Germany), centrifuge (54178; Eppendorf, Hamburg, Germany), electrophoresis apparatus DYY-2C and shaker WD-9405B both from (Beijing Liuyi Instrument Factory, Beijing, China), refrigerator (BCD256KFB; Haier Group, China), MightySmall Transphor (Amersham Biosciences Corp., Piscataway, NJ, USA), and Morris water maze (Beijing Shuolinyuan Science and Technology, Beijing, China). 
Major reagents. The major reagents used in the present study were: Sevoflurane (lot no. 11122231; Shanghai Hengrui Medicine Co., Ltd., Shanghai, China), BCA protein assay kit (PC0020; Beijing Suolaibao Science and Technology Co., Ltd., Beijing, China), skim milk powder (LP0031; Shanghai Chuanxiang Biotechnology Ltd., Shanghai, China), protein marker (801-01; Shanghai Puxin Biological Technology Co., Ltd., Henan, China), RIPA lysis buffer (C1053, Beijing Pulilai Gene Technology Co., Ltd., Beijing, China), acrylamide (AB1032; Bio Basic Inc., Ontario, Canada ), glycine (GT2065; Shanghai Biological Engineering Technology Ltd., Shanghai, China), tris (hydroxymethyl) aminomethane (TB0194; Bio Basic Inc.), 4\% paraformaldehyde in phosphate-buffered saline (PBS) solution (Shanghai Biological Engineering Company, Shanghai, China), ELISA kit for quantitative assay of mouse TNF- $\alpha$ (MTA00B), IL-6 (M6000B), and mouse IL-8 (M8000B) all from R\&D Systems, Inc., (Minneapolis, MN, USA), and Caspase 3 antibody (cat. no. ab9665; Cell Signaling Technology, Inc., Danvers, MA, USA).

Anesthesia administration. The rats were placed in the prepared anesthesia box and anaesthetized with an inhalation machine. Oxygen concentration and anesthesia doses were continuously monitored. The anesthesia time commenced when the sevoflurane concentration reached the maximum for each group. The gas flow in the anesthesia chamber was maintained at $4 \mathrm{l} / \mathrm{min}$.

Water maze test. The Morris water maze was used to test the cognitive function of the rats in each group. Since its invention in 1984, the Morris water maze has been used to evaluate the spatial learning ability and behavior of animals (6). Therefore, the Morris water maze can be used to examine the cognitive competence of animals after any intervention $(7,8)$. The rats were placed into the water at the midpoint of the pool wall in a quadrant. A platform was set in the southwest quadrant, approximately $25 \mathrm{~cm}$ from the wall. The distance from the platform to the wall was equal to that from the platform to the pool center. The temperature and light of the water maze were kept constant. The rats were trained for 1 week, 8 times a day. Each time a rat was placed into the water maze, the latent period was recorded before finding the platform for a total of $90 \mathrm{sec}$. The total length of the swimming route was recorded.

Harvest of the hippocampal tissue. After the end of the experiments, the rats were sacrificed by cervical dislocation and the whole brain was isolated and placed on tin foil paper. The cortical tissue was removed with ophthalmological forceps and the hippocampi harvested. Total protein was extracted from the hippocampus and quantified.

Western blot analysis. The total protein homogenates from hippocampal tissue were resolved in an 8-10\% gradient SDS-PAGE and transferred onto nitrocellulose membranes by semi-dry method. The membrane was blocked using blocking solution (5\% skim milk powder) and incubated at $4^{\circ} \mathrm{C}$ for $1 \mathrm{~h}$. The rabbit anti-rat caspase- 3 polyclonal primary antibody (1:5,000, cat. no. 9662; Cell Signaling Technology, Inc.) was added onto the membrane, followed by overnight incubation at $4^{\circ} \mathrm{C}$. After 4 washes in PBS, the secondary goat anti-rabbit $\operatorname{IgG}$ polyclonal antibody (1:10,000; cat. no. 7074;
Table I. Effects of sevoflurane on cognitive function of rats after anesthesia.

\begin{tabular}{lcc}
\hline Groups & $\begin{array}{c}\text { Latent period } \\
(\mathrm{sec})\end{array}$ & $\begin{array}{c}\text { Total route length } \\
(\mathrm{cm})\end{array}$ \\
\hline Control & $9.41 \pm 1.23$ & $972 \pm 85$ \\
Low-dose & $10.86 \pm 1.34^{\mathrm{a}}$ & $854 \pm 98^{\mathrm{a}}$ \\
High-dose & $16.52 \pm 3.29^{\mathrm{b}}$ & $339 \pm 101^{\mathrm{b}}$ \\
Nimodipine & $12.04 \pm 3.46^{\mathrm{c}}$ & $536 \pm 127^{\mathrm{c}}$ \\
\hline
\end{tabular}

${ }^{\mathrm{a}} \mathrm{P}<0.05$; ${ }^{\mathrm{b}} \mathrm{P}<0.01$ compared with the control group; ${ }^{\mathrm{c}} \mathrm{P}<0.01$ compared with the higher-dose group.

Cell Signaling Technology, Inc.) was added for $2 \mathrm{~h}$. The membrane was rinsed three times using sterile $4 \%$ paraformaldehyde in PBS, followed by chromogenic reaction, exposure, development, and documentation. Finally, optical density of the bands was determined by using a gel imaging system, and the protein expression quantitatively analyzed.

Statistical analysis. SPSS 19.0 software (SPSS, Inc., Chicago, IL, USA) was used for statistical analysis. Measurement data were presented as means $\pm \mathrm{SD}$, and inter-group data were compared using single factor ANOVA. $\mathrm{P}<0.05$ was considered to indicate a statistically significant difference.

\section{Results}

Effect of sevoflurane on cognitive function of rats after inhalation anesthesia. After treating rats with sevoflurane, the latent period in the Morris water maze was examined. Sevoflurane anesthesia significantly prolonged the latent period and significantly shortened the total length of the swimming route of the rats in a dose-dependent manner (Table I). After high-dose sevoflurane and nimodipine, the latent period was significantly shorter and the total length of swimming route was longer than that of the high-dose sevoflurane group (Table I). These results suggested a decreased cognitive function for rats treated with sevoflurane.

Effect of sevoflurane on IL-6/IL-8 expression. The expression of IL-6/IL-8 in hippocampus after anesthesia was examined to determine the potential mechanisms mediating the effects of sevoflurane on cognition. The western blot analysis showed that IL-6 and IL-8 were significantly upregulated in hippocampus after sevoflurane anesthesia compared with that of the control group (Fig. 1), indicating hippocampus inflammation. After nimodipine intervention the abnormal expression of IL-6 and IL-8 in rats was partially reversed compared with the high-dose group (Fig. 1).

Effect of sevoflurane on TNF- $\alpha$ expression. The activation of other inflammatory pathways were studied by examining the expression of TNF- $\alpha$. TNF- $\alpha$ was significantly upregulated in hippocampus after sevoflurane anesthesia compared with that of the control group (Fig. 2), supporting the inflammation of hippocampus. After nimodipine intervention, the TNF- $\alpha$ 

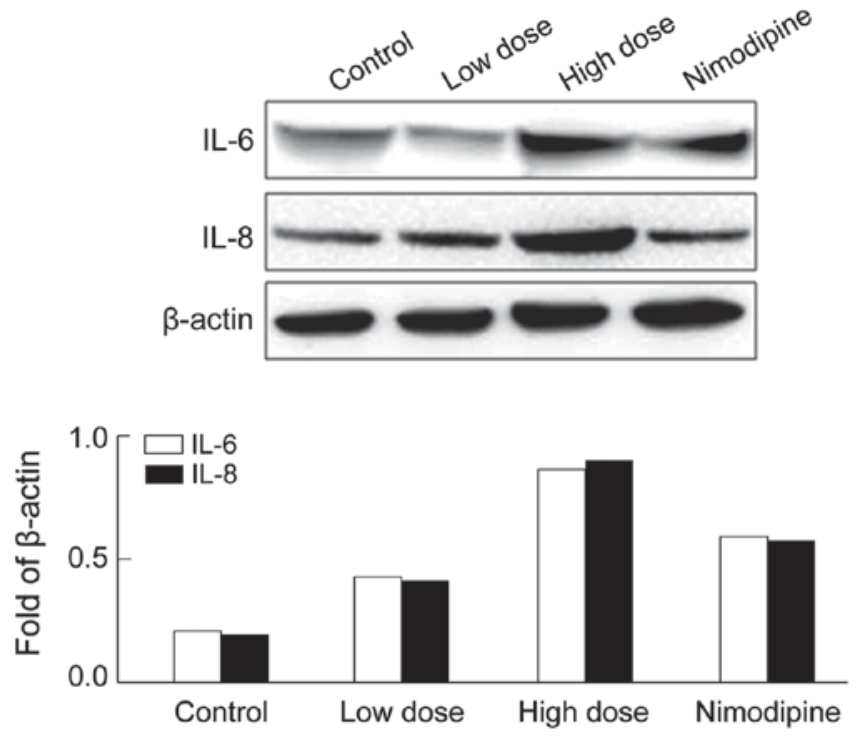

Figure 1. Effect of sevoflurane anesthesia on IL-6/IL-8 expression in rats. IL, interleukin $-6,-8$.
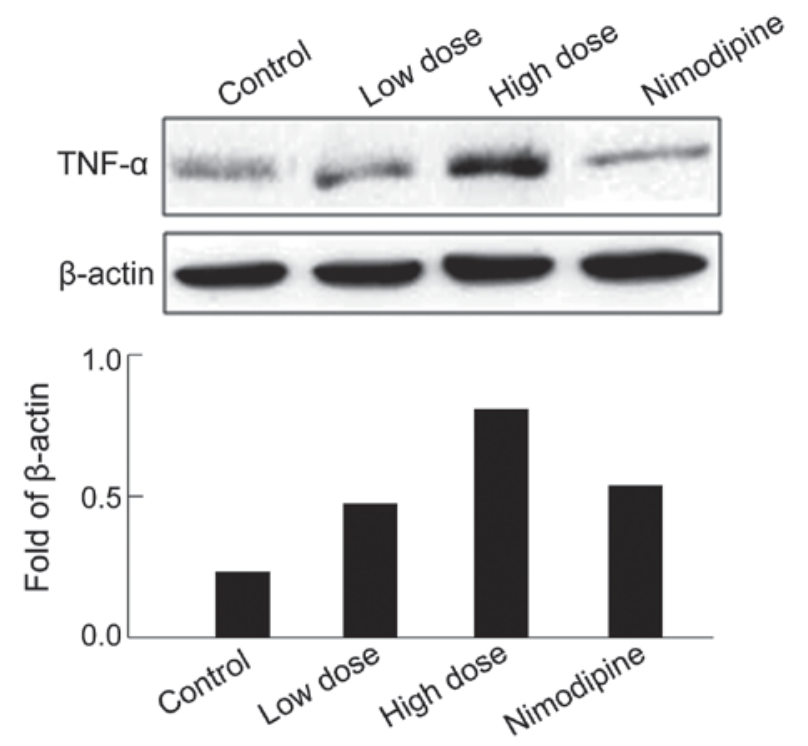

Figure 2. Effect of sevoflurane on TNF- $\alpha$ expression in rats. TNF- $\alpha$, tumour necrosis factor- $\alpha$.

expression was partially restored compared with the high-dose group (Fig. 2).

Effects of sevoflurane on Caspase-3 expression. Activation of apoptosis markers after anesthesia were also examined. The results showed that Caspase-3 expression was upregulated in rat hippocampus after sevoflurane anesthesia compared with that of the control group (Fig. 3), indicating significant neuronal death in the hippocampus. After nimodipine intervention, Caspase-3 expression was partially restored compared with that of the high-dose group (Fig. 3).

\section{Discussion}

Postoperative cognitive dysfunction causes personality disorder, decrease of social ability, loss of interpersonal skills,
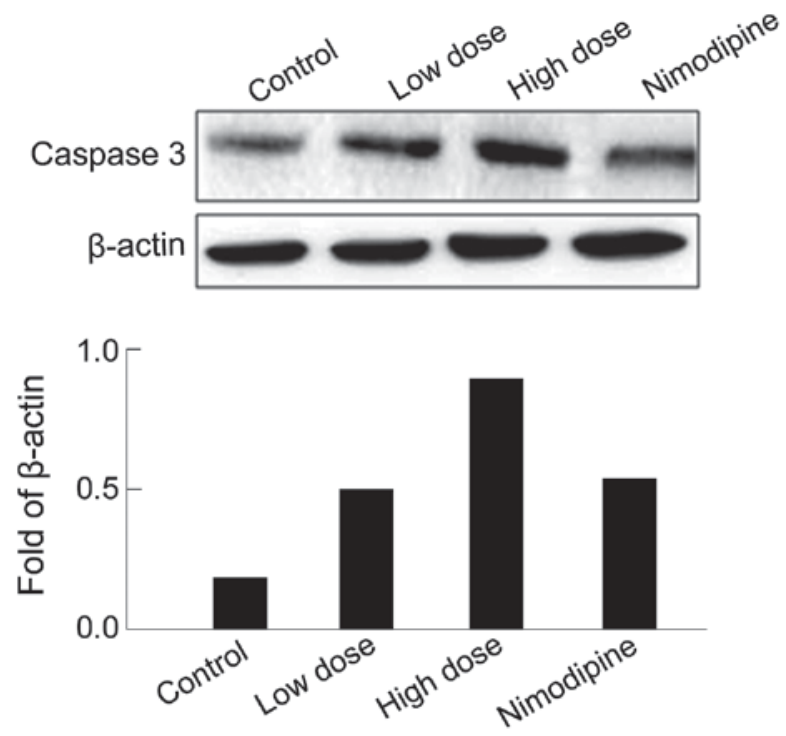

Figure 3. Effect of sevoflurane anesthesia on Caspase-3 expression in rats.

and seriously affects the quality of life because of severe hypomnesis, inattention, and other mental issues (9). The International Study of Post-Operative Cognitive Dysfunction (ISPOCD) specializes in the largest multicenter study currently to define the relationship between anesthesia and postoperative cognitive dysfunction. The ISPOCD study concluded that the incidence rate of postoperative cognitive dysfunction was $26 \%$ one week after surgery, $10 \%$ three months after surgery, and $1 \%$ two years after surgery (except for cardiac surgery) (10). During cardiac surgery when a cardiopulmonary bypass device was used, the incidence of postoperative cognitive dysfunction was $53 \%$ one week after surgery and $42 \%$ in the intervening five years. Based on these results, scholars now pay more attention to the occurrence of postoperative cognitive dysfunction.

The specific mechanisms of cognitive dysfunction caused by anesthesia remain unclear. Based on the existing theories, some possible mechanisms include: i) Anesthesia causes direct toxic effects on calcium homeostasis in neurons (11); ii) the neuroinflammation system is aggravated by surgical injury (12); iii) the physiological functions of neural stem cells in the brain are inhibited by anesthesia (13); and iv) anesthesia exacerbates the endogenous neurodegeneration processes (14). Our study confirmed that the cognitive function of rats decreased after sevoflurane anesthesia. Previous findings have suggested that sevoflurane can activate specific enzymes and cause protein denaturation (15). Additionally, sevoflurane can induce ER stress, induce apoptosis, and lead to cognitive dysfunction (16). We confirmed that sevoflurane upregulated Caspase-3 in hippocampal tissue.

Neurodegenerative diseases can cause damage to the cognitive function and inflammation plays an important role in these pathologies $(17,18)$. During surgical intervention, the stress response can lead to the production of several inflammatory factors such as TNF- $\alpha$, IL-6, and IL-8. These inflammatory factors promote the release of other inflammatory factors, thus aggravating the inflammatory response. Sevoflurane is known to increase the expression level of IL- 6 and TNF- $\alpha$, thereby causing neuroinflammation (19). In the present study, we 
confirmed that sevoflurane upregulated the levels of TNF- $\alpha$, IL-6, and IL-8 in anesthetized rats, supporting the role of neuroinflammation to the occurrence of postoperative cognitive dysfunction $(20,21)$.

Elevated intracellular calcium is a common pathway associated with nerve impairment that can be caused by multiple factors. Calcium overload is a pathological state that can induce metabolic disequilibrium, which eventually leads to cognitive dysfunction (22). Calcium overload in the brain can trigger harmful processes such as inflammation, injury, and neuronal death (23). Moreover, calcium overload in the hippocampus affects neurotransmitter release by decreased synaptic conductivity. This disturbance of synaptic transmission ultimately aggravates learning and memory dysfunction (24). Nimodipine is a dihydropyridine calcium channel blocker whose targets are the brain vessels and neurons. In the present study, we have shown that nimodipine restored neuronal function in the hippocampus after anesthesia and partially recovered the cognitive dysfunction caused by sevoflurane inhalation. Thus, the neuroprotective activity of nimodipine suggest that sevoflurane causes neuronal dysfunction, at least in part, by disrupting calcium transport (25). Furthermore, nimodipine partially restored the abnormal expression of TNF- $\alpha$, IL-6, IL-8, and Caspase-3, suggesting that neuroinflammation and cell death are pathways activated downstream of the calcium dysfunction.

In conclusion, the sevoflurane-induced cognitive dysfunction may be related to the activation of TNF- $\alpha$, IL- 6 , IL- 8 , and Caspase- 3 and other inflammatory and apoptotic factors. The administration of nimodipine can partially restore the damage caused by sevoflurane, suggesting altered calcium transport.

\section{Competing interests}

The authors declare that they have no competing interests.

\section{References}

1. Tang L, Liu H, Wu Y, Li M, Li W, Jiang M, Hou J, Jiang Y, $\mathrm{Xia} Z$ and Meng Q: Sevoflurane may be more beneficial than propofol in patients receiving endoscopic variceal ligation and endoscopic variceal sclerotherapy: A randomized, double-blind study. Exp Ther Med 14: 3145-3152, 2017.

2. Peng S, Zhang Y, Li GJ, Zhang DX, Sun DP and Fang Q: The effect of sevoflurane on the expression of M1 acetylcholine receptor in the hippocampus and cognitive function of aged rats. Mol Cell Biochem 361: 229-233, 2012.

3. Monk TG, Weldon BC, Garvan CW, Dede DE, van der Aa MT, Heilman KM and Gravenstein JS: Predictors of cognitive dysfunction after major noncardiac surgery. Anesthesiology 108: $18-30,2008$

4. Feng X, Liu JJ, Zhou X, Song FH, Yang XY, Chen XS, Huang WQ, Zhou LH and Ye JH: Single sevoflurane exposure decreases neuronal nitric oxide synthase levels in the hippocampus of developing rats. Br J Anaesth 109: 225-233, 2012.

5. Bork K, Wurm F, Haller H, Strauss C, Scheller C, Gnanapragassam VS and Horstkorte R: Neuroprotective and neuroregenerative effects of nimodipine in a model system of neuronal differentiation and neurite outgrowth. Molecules 20: 1003-1013, 2015.

6. Choeiri C, Hewitt K, Durkin J, Simard CJ, Renaud JM and Messier C: Longitudinal evaluation of memory performance and peripheral neuropathy in the Ins2 ${ }^{\mathrm{C} 96 \mathrm{Y}}$ Akita mice. Behav Brain Res 157: 31-38, 2005.
7. Fitsanakis VA, Thompson KN, Deery SE, Milatovic D, Shihabi ZK, Erikson KM, Brown RW and Aschner M: A chronic iron-deficient/high-manganese diet in rodents results in increased brain oxidative stress and behavioral deficits in the morris water maze. Neurotox Res 15: 167-178, 2009.

8. Kumar A, Seghal N, Padi SV and Naidu PS: Differential effects of cyclooxygenase inhibitors on intracerebroventricular colchicine-induced dysfunction and oxidative stress in rats. Eur J Pharmacol 551: 58-66, 2006.

9. Umholtz $M$ and Nader ND: Anesthetic immunomodulation of the neuroinflammation in postoperative cognitive dysfunction. Immunol Invest 46: 805-815, 2017.

10. Jungwirth B, Zieglgänsberger W, Kochs E and Rammes G: Anesthesia and postoperative cognitive dysfunction (POCD). Mini Rev Med Chem 9: 1568-1579, 2009.

11. Wei $\mathrm{H}$ and Xie Z: Anesthesia, calcium homeostasis and Alzheimer's disease. Curr Alzheimer Res 6: 30-35, 2009.

12. Wan Y, Xu J, Ma D, Zeng Y, Cibelli M and Maze M: Postoperative impairment of cognitive function in rats: A possible role for cytokine-mediated inflammation in the hippocampus. Anesthesiology 106: 436-443, 2007.

13. Sall JW, Stratmann G, Leong J, McKleroy W, Mason D, Shenoy S, Pleasure SJ and Bickler PE: Isoflurane inhibits growth but does not cause cell death in hippocampal neural precursor cells grown in culture. Anesthesiology 110: 826-833, 2009.

14. Bohnen NI, Warner MA, Kokmen E, Beard CM and Kurland LT: Alzheimer's disease and cumulative exposure to anesthesia: A case-control study. J Am Geriatr Soc 42: 198-201, 1994

15. Le Freche H, Brouillette J, Fernandez-Gomez FJ, Patin P, Caillierez R, Zommer N, Sergeant N, Buée-Scherrer V, Lebuffe G, Blum D and Buée L: Tau phosphorylation and sevoflurane anesthesia: An association to postoperative cognitive. Impairment Anesthesiology, 116 (4): 779-787, 2012.

16. Chen G, Gong M, Yan M and Zhang X: Sevoflurane induces endoplasmic reticulum stress mediated apoptosis in hippocampal neurons of aging rats. PLoS One 8: e57870, 2013.

17. Shih J, May LD, Gonzalez HE, Lee EW, Alvi RS, Sall JW, Rau V, Bickler PE, Lalchandani GR, Yusupova M, et al: Delayed environmental enrichment reverses sevoflurane-induced memory impairment in rats. Anesthesiology 116: 586-602, 2012.

18. Yang HK, Chungh DS and Hwang JM: The effect of general anesthesia and strabismus surgery on the intellectual abilities of children: A pilot study. Am J Ophthalmol 153: 609-613, 2012.

19. Zhang L, Zhang J, Yang L, Dong Y, Zhang Y and Xie Z: Isoflurane and sevoflurane increase interleukin-6 levels through the nuclear factor-kappa B pathway in neuroglioma cells. Br J Anaesth 110 (Suppl 1): 82-91, 2013.

20. Kronfol $Z$ and Remick DG: Cytokines and the brain: Implications for clinical psychiatry. Am J Psychiatry 157: 683-694, 2000.

21. Akiyama H, Barger S, Barnum S, Bradt B, Bauer J, Cole GM, Cooper NR, Eikelenboom P, Emmerling M, Fiebich BL, et al: Inflammation and Alzheimer's disease. Neurobiol Aging 21: 383-421, 2000.

22. Kandel ER, Schwartz JH and Jessell TM: Principles of neuronal science (4th Edition). McGraw-Hill, New York, NY, pp386-409, 2001.

23. Vannucci RC, Brucklacher RM and Vannucci SJ: Intracellular calcium accumulation during the evolution of hypoxic-ischemic brain damage in the immature rat. Brain Res Dev Brain Res 126: $117-120,2001$.

24. Foster TC and Norris CM: Age-associated changes in $\mathrm{Ca}^{2+}{ }^{2+}$ dependent processes: Relation to hippocampal synaptic plasticity. Hippocampus 7: 602-612, 1997.

25. Macdonald RL and Kelly KM: Antiepileptic drug mechanisms of action. Epilepsia 36 (Suppl 2): 2-12, 1995.

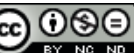

This work is licensed under a Creative Commons Attribution-NonCommercial-NoDerivatives 4.0 International (CC BY-NC-ND 4.0) License. 